\title{
Three-valued matrices with classical consequence relation for an arbitrary propositional language
}

\author{
L. Yu. DEVYATKIN
}

\begin{abstract}
In this paper the class of three-valued matrices with classical consequence relations is defined.

Keywords: classical propositional logic, three-valued logics, consequence relation
\end{abstract}

The aim of this paper is to determine what properties the basic functions of an arbitrary three-valued logical matrix must possess to make the consequence relation determined by that matrix classical. Let us begin with defining the necessary basic notions.

A logical matrix is a structure $\mathfrak{M}=\langle V, F, D\rangle$, where $V$ is the set of truth-values, $F$ is a set of functions on $V$ called basic functions, and $D$ is a designated subset of $V$.

Let us consider an arbitrary propositional language $L$. A matrix $\mathfrak{M}$ is a matrix for $L$ iff there is a one-to-one correspondence between the elements of $F$ and the connectives of $L$. If such a correspondence exists we can define a valuation of an $L$-formula in $\mathfrak{M}$.

A valuation $v$ of formula $A$ in $\mathfrak{M}$ is a homomorphism of $L$ in $<V, F>$ such that

1. if $A$ is a propositional variable, then $v(A) \in V$;

2. if $A_{1}, A_{2}, \cdots, A_{n}$ are formulas, and $\otimes$ is an $n$-ary connective of $L$, then $v\left(\otimes\left(A_{1}, A_{2}, \cdots, A_{n}\right)\right)=f^{n}\left(v\left(A_{1}\right), v\left(A_{2}\right), \cdots, v\left(A_{n}\right)\right)$, where $f^{n}$ is a function from $F$ corresponding to $\otimes$. 
Subsequently, we can define the consequence relation in $\mathfrak{M}$ in a standard way.

$\Gamma \vDash(\mathfrak{M}) B$ iff there is no valuation $v$ in $\mathfrak{M}$, such that $v[\Gamma] \subseteq D(\mathfrak{M})$ (i.e. every formula from $\Gamma$ assumes a truth-value designated in $\mathfrak{M}$ ), and $v(A) \notin D(\mathfrak{M})$.

Let us denote as $\vDash(\mathfrak{M})$ a set of ordered pairs $\langle\Gamma, B\rangle$, such that $\Gamma$ is a set of formulas, $B$ is a formula, and $\Gamma \vDash(\mathfrak{M}) B$.

We will say that the consequence relation in some threevalued matrix $\mathfrak{N}$ for $L$ is classical iff there is a matrix $\mathfrak{M}_{C}=<\{1,0\}, F\left(\mathfrak{M}_{C}\right),\{0\}>$ for $L$, where $F\left(\mathfrak{M}_{C}\right)$ is functionally complete in $P_{2}$, and $\vDash\left(\mathfrak{M}_{C}\right)=\vDash(\mathfrak{N})$.

Because $F\left(\mathfrak{M}_{C}\right)$ is complete in $P_{2}$, the following functions can be presented as compositions of some elements of $F\left(\mathfrak{M}_{C}\right)$ :

- $f_{\wedge}(x, y)=1$ iff $x=1$ and $y=1$;

- $f_{\neg}(x)=1$ iff $x=0$.

Actually, there can be more than one composition for $f_{\wedge}(x, y)$ or $f_{\neg}(x)$. But for our purposes it is only essential that we pick one particular composition for each function. Then we can make the following reasoning.

As there is a one-to-one correspondence between the functions of $F\left(\mathfrak{M}_{C}\right)$ and the connectives of $L$, there is one particular $L$-formula $F_{\wedge}$ corresponding to $f_{\wedge}(x, y)$ and one particular $L$-formula $F_{\neg}$ corresponding to $f_{\neg}(x)$. Because $\mathfrak{N}$ is a matrix for $L$ and therefore there is a one-to-one correspondence between the functions of $F(\mathfrak{N})$ and the connectives of $L$, there is one particular composition $g_{\wedge}(x, y)$ of the elements of $F(\mathfrak{N})$ corresponding to $L$-formula $F_{\wedge}$ and one particular composition $g_{\neg}(x)$ of the elements of $F(\mathfrak{N})$ corresponding to $L$-formula $F_{\neg}$.

Let us use $\wedge$ and $\neg$ as abbreviations for $F_{\wedge}$ and $F_{\neg}$. If $\operatorname{Form}(L)$ is the set of all formulas of $L$, then $\operatorname{Form}_{\wedge, \neg}(L)$ will be its subset which consists exclusively of the formulas not including any connectives but $\wedge$ and $\neg$. We will denote the elements of Form $_{\wedge, \neg}(L)$ as $L_{\wedge, \neg}$-formulas. As Form $_{\wedge, \neg}(L)$ is a subset of $\operatorname{Form}(L)$, the following is true. 
ObSERVATION 1. If for every set of $L$-formulas $\Gamma$ and for every $L$ formula $B$ it is true that $\Gamma \vDash(\mathfrak{N}) B$ iff $\Gamma \vDash\left(\mathfrak{M}_{C}\right) B$, then for every set of $L_{\wedge, \neg}$-formulas $\Gamma^{*}$ and for every $L_{\wedge, \neg}$-formula $B^{*}$ it is true that $\Gamma^{*} \vDash(\mathfrak{N}) B^{*}$ iff $\Gamma^{*} \vDash\left(\mathfrak{M}_{C}\right) B^{*}$.

On the basis of this Observation 1 we can propose the following Lemma 1 concerning the properties of $g_{\wedge}(x, y)$ or $g_{\neg}(x)$.

LEmma 1. If for every set of $L$-formulas $\Gamma$ and for every $L$-formula $B$ it is true that $\Gamma \vDash(\mathfrak{N}) B$ iff $\Gamma \vDash\left(\mathfrak{M}_{C}\right) B$, then

- $g_{\wedge}(x, y) \in D(\mathfrak{N})$ iff $x \in D(\mathfrak{N})$ and $y \in D(\mathfrak{N})$;

- $g_{\neg}(x) \in D(\mathfrak{N})$ iff $x \notin D(\mathfrak{N})$.

PRoOF. We will begin with proving the first part. Let us suppose that for some $x^{\prime}$ and $y^{\prime}$ it is true that $g_{\wedge}\left(x^{\prime}, y^{\prime}\right) \in D(\mathfrak{N})$ and $x^{\prime} \notin D(\mathfrak{N})$. Let $p_{1}$ and $p_{2}$ be the propositional variables of $L$ and $v^{\prime}$ be such a valuation in $\mathfrak{N}$ that $v^{\prime}\left(p_{1}\right)=x^{\prime}$ and $v^{\prime}\left(p_{2}\right)=y^{\prime}$. By definition of valuation, $v^{\prime}\left(p_{1} \wedge p_{2}\right)=g_{\wedge}\left(x^{\prime}, y^{\prime}\right)$. So $v^{\prime}$ is such a valuation in $\mathfrak{N}$ that $v^{\prime}\left(p_{1} \wedge p_{2}\right) \in D(\mathfrak{N})$ and $v^{\prime}\left(p_{1}\right) \notin D(\mathfrak{N})$. This entails, by definition of $\vDash$, that $p_{1} \wedge p_{2} \not \models(\mathfrak{N}) p_{1}$. But $p_{1} \wedge p_{2} \vDash\left(\mathfrak{M}_{C}\right) p_{1}$, and $p_{1} \wedge p_{2}, p_{1}$ are $L_{\wedge, \neg}$-formulas. So we come to the contradiction with Observation 1. The reasoning for $y^{\prime}$ is analogous.

Now let us suppose that for some $x^{\prime}$ and $y^{\prime}$ it is true that $g_{\wedge}\left(x^{\prime}, y^{\prime}\right) \notin D(\mathfrak{N})$, and $x^{\prime} \in D(\mathfrak{N})$ and $y^{\prime} \in D(\mathfrak{N})$. Again, let $v^{\prime}$ be such a valuation in $\mathfrak{N}$ that $v^{\prime}\left(p_{1}\right)=x^{\prime}$ and $v^{\prime}\left(p_{2}\right)=y^{\prime}$. Then $v^{\prime}$ is such a valuation in $\mathfrak{N}$ that $v^{\prime}\left(p_{1} \wedge p_{2}\right) \notin D(\mathfrak{N})$, and $v^{\prime}\left(p_{1}\right) \in D(\mathfrak{N})$ and $v^{\prime}\left(p_{2}\right) \in D(\mathfrak{N})$. From this, by definition of $\vDash$, it follows that $p_{1}, p_{2} \not \models(\mathfrak{N}) p_{1} \wedge p_{2}$. But $p_{1}, p_{2} \vDash\left(\mathfrak{M}_{C}\right) p_{1} \wedge p_{2}$, and either $p_{1} \wedge p_{2}$, $p_{1}$ and $p_{2}$ are $L_{\wedge, \neg}$-formulas. Therefore our assumption contradicts Observation 1. The proof of the first statement is complete.

Let us prove the second statement of the lemma. First, we will assume that there is some $x^{\prime}$, such that $g_{\neg}\left(x^{\prime}\right) \in D(\mathfrak{N})$ and $x^{\prime} \in$ $D(\mathfrak{N})$. As propositional variables are the elements of $\operatorname{Form}_{\wedge, \neg}(L)$, due to Observation 1 and the fact that $p_{1}, \neg p_{1} \vDash\left(\mathfrak{M}_{C}\right) p_{2}$, it is necessary that $p_{1}, \neg p_{1} \vDash(\mathfrak{N}) p_{2}$. Now let us consider a valuation $v^{\prime}$, such that $v^{\prime}\left(p_{1}\right)=x^{\prime}, v^{\prime}\left(p_{2}\right)=y^{\prime}$ and $y^{\prime} \notin D(\mathfrak{N})$. By definition of valuation and by our assumption, $v^{\prime}\left(\neg p_{1}\right) \in D(\mathfrak{N})$. According to 
the definition of $\vDash$, it means that $p_{1}, \neg p_{1} \not \models(\mathfrak{N}) p_{2}$, and we arrive to the contradiction.

Now let us assume that there is some $x^{\prime}$, such that $g_{\neg}\left(x^{\prime}\right) \notin D(\mathfrak{N})$ and $x^{\prime} \notin D(\mathfrak{N})$. As $p_{2} \vDash\left(\mathfrak{M}_{C}\right) \neg\left(p_{1} \wedge \neg p_{1}\right)$, it is necessary that $p_{2} \vDash(\mathfrak{N}) \neg\left(p_{1} \wedge \neg p_{1}\right)$. Therefore, $g_{\neg}\left(g_{\wedge}\left(x^{\prime}, g_{\neg}\left(x^{\prime}\right)\right)\right) \in D(\mathfrak{N})$. We have shown above that, if $g_{\neg}(x) \in D(\mathfrak{N})$, then $x \notin D(\mathfrak{N})$. So $g_{\wedge}\left(x^{\prime}, g_{\neg}\left(x^{\prime}\right)\right) \notin D(\mathfrak{N})$. At the same time $g_{\wedge}\left(x^{\prime}, g_{\neg}\left(x^{\prime}\right)\right) \neq x^{\prime}$, as $g_{\neg}\left(x^{\prime}\right) \notin D(\mathfrak{N})$, and we have already shown that $g_{\wedge}(x, y) \in D(\mathfrak{N})$ iff $x \in D(\mathfrak{N})$ and $y \in D(\mathfrak{N})$. This entails that there must exist a value $x^{\prime \prime}$, such that $x^{\prime \prime} \notin D(\mathfrak{N})$ and $g_{\neg}\left(x^{\prime \prime}\right) \in D(\mathfrak{N})$. We have only three elements in $V(\mathfrak{N})$, and there must be at least one element in $D(\mathfrak{N})$. Otherwise, it would be impossible that $\vDash\left(\mathfrak{M}_{C}\right)=\vDash(\mathfrak{N})$. Consequently, either $g_{\neg}\left(x^{\prime}\right)=x^{\prime \prime}$ or $g_{\neg}\left(x^{\prime}\right)=x^{\prime}$. If $g_{\neg}\left(x^{\prime}\right)=x^{\prime \prime}$, then $g_{\neg}\left(g_{\neg}\left(x^{\prime}\right)\right) \in D(\mathfrak{N})$. Let us consider a valuation $v^{\prime}$, such that $v^{\prime}\left(p_{1}\right)=x^{\prime}$. We have that $v^{\prime}\left(p_{1}\right) \notin D(\mathfrak{N})$ and $v^{\prime}\left(\neg \neg p_{1}\right) \in D(\mathfrak{N})$. Thus, $\neg \neg p_{1} \not \models(\mathfrak{N}) p_{1}$. But that contradicts the assumption that $\vDash\left(\mathfrak{M}_{C}\right)=\vDash(\mathfrak{N})$ as $\neg \neg p_{1} \vDash\left(\mathfrak{M}_{C}\right) p_{1}$. So $g_{\neg}\left(x^{\prime}\right)=x^{\prime}$ and, as a consequence, $g_{\neg}\left(g_{\neg}\left(x^{\prime}\right)\right)=x^{\prime}$. Then $g_{\neg}\left(g_{\wedge}\left(x^{\prime}, g_{\neg}\left(g_{\neg}\left(x^{\prime}\right)\right)\right)\right) \in$ $D(\mathfrak{N})$. Now let us consider the valuation $v^{\prime}$ as defined above. We have that $v^{\prime}\left(\neg p_{1}\right) \notin D(\mathfrak{N})$ and $v^{\prime}\left(\neg\left(p_{1} \wedge \neg \neg p_{1}\right)\right) \in D(\mathfrak{N})$, so $\neg\left(p_{1} \wedge \neg \neg p_{1}\right) \not \models(\mathfrak{N}) \neg p_{1}$. Again, we arrive at the contradiction, because $\neg\left(p_{1} \wedge \neg \neg p_{1}\right) \vDash\left(\mathfrak{M}_{C}\right) \neg p_{1}$. This concludes the proof of our lemma.

Now we will define the necessary and sufficient properties that $\mathfrak{N}$ must possess if $\vDash\left(\mathfrak{M}_{C}\right)=\vDash(\mathfrak{N})$. The method that we use is a generalization of the idea presented in [1].

DeFinition 1. If $v$ is a valuation in $\mathfrak{N}$, then $\bar{v}$ is such a valuation in $\mathfrak{M}_{C}$ that $\bar{v}(p)=1$ iff $v(p) \in D(\mathfrak{N})$ and $\bar{v}(p)=0$ iff $v(p) \notin D(\mathfrak{N})$.

Definition 2. $h_{1,0}$ is a homomorphism of $V(\mathfrak{N})$ on $V\left(\mathfrak{M}_{C}\right)$, such that $h_{1,0}(x)=1$ iff $x \in D(\mathfrak{N})$ and $h_{1,0}(x)=0$ iff $x \notin D(\mathfrak{N})$.

Theorem 1. $\vDash\left(\mathfrak{M}_{C}\right)=\vDash(\mathfrak{N})$ iff for every $v$ and every formula $A$ it is true that $h_{1,0}(v(A))=\bar{v}(A)$.

Proof. First we will prove that if $\vDash\left(\mathfrak{M}_{C}\right)=\vDash(\mathfrak{N})$, then for every $v$ and every formula $A$ it is true that $h_{1,0}(v(A))=\bar{v}(A)$. Let us 
suppose that $\vDash\left(\mathfrak{M}_{C}\right)=\vDash(\mathfrak{N})$ and $h_{1,0}(v(A)) \neq \bar{v}(A)$ for some $v$ and $A$.

Due to the fact that $\left\{f_{\wedge}(x, y), f_{\neg}(x)\right\}$ is complete in $P_{2}$, for every $L$-formula $A$ there is an $L_{\wedge, \neg}-$ formula $A^{*}$ such that $u(A)=u\left(A^{*}\right)$ for every valuation $u$ in $\mathfrak{M}_{C}$. Consequently, $A \vDash\left(\mathfrak{M}_{C}\right) A^{*}$ and $A^{*} \vDash\left(\mathfrak{M}_{C}\right) A$. If $\vDash\left(\mathfrak{M}_{C}\right)=\vDash(\mathfrak{N})$, it is also true that $A \vDash(\mathfrak{N}) A^{*}$ and $A^{*} \vDash(\mathfrak{N}) A$. By definition of $\vDash$, we have that in this case for every valuation $v$ in $\mathfrak{N}$ it is true that $v(A) \in D(\mathfrak{N})$ iff $v\left(A^{*}\right) \in D(\mathfrak{N})$. Therefore for every $A$ and every $v$ if $h_{1,0}(v(A)) \neq \bar{v}(A)$, then there is an $L_{\wedge, \neg}$-formula $A^{*}$ such that $h_{1,0}\left(v\left(A^{*}\right)\right) \neq \bar{v}\left(A^{*}\right)$. Let us consider such $A^{*}$ that it is $L_{\wedge, \neg}$-formula with the least amount of instances of $\wedge$ and $\neg$ for which $h_{1,0}\left(v\left(A^{*}\right)\right) \neq \bar{v}\left(A^{*}\right)$.

According to the definitions of $\bar{v}$ and $h_{1,0}, \bar{v}(p)=h_{1,0}(v(p))$. So $A^{*}$ is either $\neg B$ or $C \wedge D$.

Let us assume that $A^{*}$ is $\neg B$. Either $h_{1,0}(v(\neg B))=1$ and $\bar{v}(\neg B)=0$ or $h_{1,0}(v(\neg B))=0$ and $\bar{v}(\neg B)=1$. Let us assume the former.

If $h_{1,0}(v(\neg B))=1$, then $v(\neg B) \in D(\mathfrak{N})$. By the definition of valuation $v(\neg B)=g_{\neg} v(B)$. By Lemma 1 , if $g_{\neg}(x) \in D(\mathfrak{N})$, then $x \notin D(\mathfrak{N})$. So $v(B) \notin D(\mathfrak{N})$. Then $h_{1,0}(v(B))=0$. As $B$ contains less connectives then $A^{*}, h_{1,0}(v(B))=\bar{v}(B)$ and $\bar{v}(B)=0$. By the definition of $f_{\neg}, f_{\neg}(\bar{v}(B))=1$. Thus, by the definition of valuation, $\bar{v}(\neg B))=1$. But that contradicts to our assumption that $\bar{v}(\neg B)=0$.

If we assume that $h_{1,0}(v(\neg B))=0$ and $\bar{v}(\neg B)=1$, the reasoning will be analogous. If $h_{1,0}(v(\neg B))=0$, then $v(\neg B) \notin D(\mathfrak{N})$, and, by Lemma $1, v(B) \in D(\mathfrak{N})$. From this we infer $h_{1,0}(v(B))=\bar{v}(B)=1$, which entails that $\bar{v}(\neg B)=0$. Again, we have a contradiction. So $A^{*}$ is not $\neg B$.

Let us assume that $A^{*}$ is $C \wedge D$. Either $h_{1,0}(v(C \wedge D))=1$ and $\bar{v}(C \wedge D)=0$ or $h_{1,0}(v(C \wedge D))=0$ and $\bar{v}(C \wedge D)=1$. Let us assume the former.

By the definition of valuation, $v(C \wedge D)=f_{\wedge}(v(C), v(D))$. By Lemma $1, g_{\wedge}(x, y) \in D(\mathfrak{N})$ iff $x \in D(\mathfrak{N})$ and $y \in D(\mathfrak{N})$. Therefore, both $v(C) \in D(\mathfrak{N})$ and $v(D) \in D(\mathfrak{N})$. As $C$ and $D$ contain less connectives than $A^{*}, h_{1,0}(v(C))=\bar{v}(C)$ and $h_{1,0}(v(D))=\bar{v}(D)$. Consequently, $\bar{v}(C)=1$ and $\bar{v}(D)=1$. By definition of $f_{\wedge}$, if 
$x=1$ and $y=1$, then $g_{\wedge}(x, y)=1$. Therefore, $f_{\wedge}(\bar{v}(C), \bar{v}(D))=1$, and, by definition of valuation, $\bar{v}(C \wedge D)=1$. Then we have a contradiction, as we have assumed that $\bar{v}(C \wedge D)=0$.

Now let us assume that $h_{1,0}(v(C \wedge D))=0$ and $\bar{v}(C \wedge D)=1$. Then we have that $v(C \wedge D) \notin D(\mathfrak{N})$. By definition of valuation, $(v(C \wedge D))=g_{\wedge}(v(C), v(D))$. By Lemma $1, g_{\wedge}(x, y) \notin D(\mathfrak{N})$ iff either $x \notin D(\mathfrak{N})$ or $y \notin D(\mathfrak{N})$. So $v(C) \notin D(\mathfrak{N})$ or $v(D) \notin D(\mathfrak{N})$. Let us assume that $v(C) \notin D(\mathfrak{N})$. Then $h_{1,0}(v(C))=0$. As $C$ contains less connectives than $A^{*}, h_{1,0}(v(C))=\bar{v}(C)$ and $\bar{v}(C)=0$. By definition of $f_{\wedge}$, if $x=0$, then $g_{\wedge}(x, y)=0$. By the definition of valuation, it follows that $\bar{v}(C \wedge D)=0$. Again, we have a contradiction, and $A^{*}$ is not $C \wedge D$.

With the reasoning above we have shown that if $\vDash\left(\mathfrak{M}_{C}\right)=\vDash(\mathfrak{N})$ no $A^{*}$ exists, for which $h_{1,0}\left(v\left(A^{*}\right)\right) \neq \bar{v}\left(A^{*}\right)$. Consequently, no such $L$-formula $A$ exists either. Now we need to prove that if for every $v$ and every formula $A$ it is true that $h_{1,0}(v(A))=\bar{v}(A)$, then $\vDash\left(\mathfrak{M}_{C}\right)=\vDash(\mathfrak{N})$.

Let us assume that $h_{1,0}(v(A))=\bar{v}(A)$ and $\vDash\left(\mathfrak{M}_{C}\right) \neq \vDash(\mathfrak{N})$. Then there exist some set of formulas $\Gamma$ and formula $B$, such that either $\Gamma \vDash\left(\mathfrak{M}_{C}\right)$ and $\Gamma \not \models(\mathfrak{N})$, or $\Gamma \not \models\left(\mathfrak{M}_{C}\right)$ and $\Gamma \vDash(\mathfrak{N})$.

Let us assume that $\Gamma \vDash\left(\mathfrak{M}_{C}\right)$ and $\Gamma \not \models(\mathfrak{N})$. Then there is some valuation $w$ in $\mathfrak{N}$, such that $w[\Gamma] \in D(\mathfrak{N})$ and $w(B) \notin D(\mathfrak{N})$. As for every valuation $v$ and every formula $A$ it is true that $h_{1,0}(v(A))=$ $\bar{v}(A)$, it is also true that $h_{1,0}\left(w\left(A_{i}\right)\right)=\bar{w}\left(A_{i}\right)$ for every element of $\Gamma$, and $h_{1,0}(w(B))=\bar{w}(B)$. Therefore, $\bar{w}$ is such a valuation in $\mathfrak{M}_{C}$ that $\bar{w}[\Gamma]=1$ and $\bar{w}(B)=0$. That means, by definition of $\vDash$, that $\Gamma \not \models\left(\mathfrak{M}_{C}\right) B$, which contradicts our assumption.

Now we will assume that $\Gamma \nvdash\left(\mathfrak{M}_{C}\right)$ and $\Gamma \vDash(\mathfrak{N})$. Then there is some valuation $u$ in $\mathfrak{M}_{C}$, such that $u[\Gamma]=1$ and $u(B)=0$. Let us construct the following valuation $w$ in $\mathfrak{N}$. For every propositional variable $p_{i}$ if $u\left(p_{i}\right)=1$, then $w\left(p_{i}\right) \in D(\mathfrak{N})$, and if $u\left(p_{i}\right)=0$, then $w\left(p_{i}\right) \notin D(\mathfrak{N})$. Obviously, for every formula $A$ it is true that $\bar{w}(A)=u(A)$. So we have that $\bar{w}[\Gamma]=1$ and $\bar{w}(B)=0$. As for every valuation $v$ and every formula $A$ it is true that $h_{1,0}(v(A))=\bar{v}(A)$, it is also true that $h_{1,0}\left(w\left(A_{i}\right)\right)=\bar{w}\left(A_{i}\right)$ for every element of $\Gamma$, and $h_{1,0}(w(B))=\bar{w}(B)$. By definition of $h_{1,0}$, it means that $w$ is such a valuation in $\mathfrak{N}$ that $w[\Gamma] \in D(\mathfrak{N})$ and $w(B) \notin D(\mathfrak{N})$. By definition 
of $\vDash$, we obtain that $\Gamma \not \models(\mathfrak{N}) B$. Again, we have arrived to the contradiction with our assumption. This concludes the proof of our theorem.

Corollary 1. Consequence relation $\vDash(\mathfrak{N})$ in a three-valued matrix $\mathfrak{N}$ for an arbitrary propositional language $L$ is classical iff for every basic operation $g_{i}^{n}\left(x_{1}, x_{2}, \ldots, x_{n}\right)$ of $\mathfrak{N}$ and the corresponding basic operation of $\mathfrak{M}_{C} f_{i}^{n}\left(y_{1}, y_{2}, \ldots, y_{n}\right)$ it is true that $g_{i}^{n}\left(x_{1}, x_{2}, \ldots, x_{n}\right) \in D(\mathfrak{N})$ if and only if $f_{i}^{n}\left(h_{1,0}\left(x_{1}\right), h_{1,0}\left(x_{2}\right), \ldots, h_{1,0}\left(x_{n}\right)\right)=1$.

\section{References}

[1] Devjatkin L.Ju., Karpenko A.S., Popov V.M. Trehznachnye harakteristicheskie matricy klassicheskoj propozicional'noj logiki // Trudy nauchno-issledovatel'skogo seminara Logicheskogo centra Instituta filosofii RAN. 2007. № 18. P.50-62 